exists. It is aimed at graduate students and professional investigators, especially those entering the field of molecular biology from other disciplines. The first part is indeed a joy, with lucid expositions of basic facts and ideas of molecular biology which surpass those in more explicitly educational books. The professional investigator, however, demands more than this, particularly where current problems and research are concerned, and it is here that the book's limitations are seen. Dr Drake's reputation as a geneticist rests primarily on his work with bacteriophage $\mathbf{T} 4$. The fact that he has naturally chosen to concentrate on this organism is both the strength and weakness of his book. The importance of T4 is obvious in those sections dealing with the principles of molecular genetics. $\mathrm{T} 4$ is, however, an organism largely independent of the cellular functions of its host and the ways in which mutations arise in it are often different from those of bacteria and even most other bacteriophages, particularly with regard to the involvement of repair and recombination processes. The connexion between recombination genes and mutation in bacteria has been a profitable field for several years and the scant attention which it receives exemplifies the book's relative neglect of the involvement of cellular processes in mutagenesis.

There are several errors (for example, in the report of the work of Kada and Mareovich on p. 161) and omissions (for example, the discoverers of the potent mutagenic action of nitrosoguanidine, Mandell and Greenberg in 1961). Nevertheless, it is a book to be commended as a reasonably elementary guide to mutation, particularly for first degree students in their final year and predoctoral workers. An interest in mutation is not out of place in a world which man is making more mutagenic than at any time since the ozone layer began to shield it from the worst effects of the Sun's radiation.

Bryn Bridges

\section{MACROMOLECULES AND GENES}

\section{The Molecular Basis of Gene Expression}

By Benjamin M. Lewin. Pp. $\mathrm{x}+446$. (Wiley (Interscience): London and New York, August 1970.) 130s boards; $70 s$ paper.

WITHIN the past decade important advances in microbial genetics, protein biosynthesis and nucleic acid biochemistry have greatly enhanced our understanding of the functioning of the genetic material, particularly in relation to control mechanisms in prokaryotic cells. As those involved in teaching or research in this field will be well aware, the rate of progress has been so rapid that it is difficult to keep fully abreast of new discoveries and their significance. The task of integrating recent developments into a coherent body of knowledge is therefore both formidable and important.

This account by Dr Lewin of the interrelationships of macromolecules involved in gene function is a timely and welcome addition to the comparatively few books in this important field. The scope of the book has been deliberately restricted chiefly to bacterial systems, which is reasonable because relatively less is known about the expression of genetic information in higher organisms. It is a pity, however, that the discussion of the control of the synthesis of multi-chain proteins such as haemoglobin and immunoglobulin is not a little more detailed.

Many of the recent developments discussed in this book are based on experimental work which is often highly sophisticated both conceptually and technically. On the whole, the presentation of intrinsically complicated material is clear and intelligible, but a good background knowledge is an advantage if not essential at times. For this reason, the book is most suitable for postgraduate and advanced undergraduate students, and for research workers active in this area. A glossary of technical terms would be of help to the reader, and perhaps this can be added in a future edition. Another aspect which could with advantage be covered more fully is the molecular basis of mutations.

There are few major errors, but the description on p. 93 of the procedure used by Dintzis for investigating the sequential synthesis of haemoglobin peptides is not entirely accurate; ribosomes in eukaryotic cells are not always attached to endoplasmic reticulum as suggested on p. 96 ; and the mechanism of action of puromycin is rather different from that which appears to be implied on p. 135. Several minor mistakes, for example the numbering of the pyrimidine ring (p. 25 and Fig. 2. 1), the spelling of several authors' names ( $p p$. 171-173). the concentration of magnesium at which ribosomes dissociate into subparticles, which is much lower than that given on p. 96, will, I hope, also be corrected on a future occasion.

On the whole, however, this is a well written and worthwhile book which will serve a useful purpose and it can be highly recommended.

H. R. V. Arnstein

\section{CONJUGATION MECHANISMS}

\section{Metabolic Conjugation and Metabolic Hydrolysis}

Vol. 1. Edited by William H. Fishman. Pp. xv +461 . (Academic: New York and Iondon, August 1970.) $261 \mathrm{~s}$.

MANY organic compounds administered to animals are excreted in the urine or bile in conjugated form and it was long thought that this is largely a process of detoxi. cation. There is now evidence, however, that hormones and other bodily constituents are likewise coupled with glucuronic acid, sulphate or other groups, so that the term "metabolic conjugation" has been introduced. The view has developed that a balance between conjugation in certain cells and cellular compartments and hydrolysis in others has important effects on the physiological and pharmacological activity of hormones and drugs. This is the first of three volumes devoted to an authoritative account of these reactions.

R. L. Smith and R. T. Williams describe the history of the discovery of conjugation mechanisms. Although this information has been available elsewhere, notably in Williams's book, Detoxication Mechanisms (Chapman and Hall), it is here conveniently summarized. D. S. Lane describes new metabolic conjugates of steroids, including coupling with $\mathrm{N}$-acetylglucosamine and glucose, acetylation, phosphorylation, methylation and combination with glutathione. Variant forms of glucuronic and sulphuric acid conjugation also remind us that biological reactions are seldom simple. C. C. Irving discusses conjugation of $\mathrm{N}$-hydroxy compounds which are of special interest in cancer induction. It is often thought that conjugated hormones are biologically inactive, but M. Mason summarizes work showing that conjugated steroids can bind with and affect the function of enzymes and other proteins. A comprehensive review of the glucuronic pathway of conjugation is given by T. A. Miettinen and E. Leskinen, and sulphoconjugation and sulphohydrolysis are described by K. S. Dodgson and F. A. Rose. The lysosomal localization of many intracellular enzymes that can hydrolyse mucopolysaccharides is reviewed by E. A. Davidson. Although much useful information has accumulated, there are unsolved problems such as the nature of the enzymes breaking down dermatan or keratan sulphates and the exact defects in storage diseases such as Hurler's and Hunter's syndromes. The final chapter by J. L. van 